\title{
Genetic Transformation of the Synaptic Pattern of a Motoneuron Class in Caenorhabditis elegans
}

\author{
W. W. Walthall and Jeffery A. Plunkett \\ Department of Biology, Georgia State University, Atlanta, Georgia 30302-4010
}

\begin{abstract}
Caenorhabditis elegans possesses two classes of inhibitory locomotory neurons, the DD and VD motoneurons (mns), and they form complementary components of a cross-inhibitory neuronal network innervating dorsal and ventral body muscles. The DD and VD mns (collectively called the D mns) share a number of morphological and neurochemical features, and mutations in a number of different genes disrupt both cell types in identical ways; however, the DD and VD mns have different lineal origins and different synaptic patterns. Given the number of phenotypic features shared by the $D$ mns, it was of interest to determine what is responsible for the synaptic patterns that distinguish them. An analysis of the locomotory defect along with a genetic epistasis test suggested that unc-55 mutations alter the function of the VD but not the DD mns. Correlated with the defective locomotory behavior of unc-55 mutants was an alteration in the distribution of varicosities, structures associated with presynaptic elements, on the VD mns. The pattern of varicosities of the unc-55 VD mns resembled that of the wild-type DD mns. Moreover, the selective removal of the DD mns revealed that unc-55 VD mns had adopted a functional role appropriate for the DD mns. Thus, unc-55 appears to be involved in producing the synaptic patterns that distinguish the two $D$ mn classes from one another; when the gene is mutated the VD and DD mns become structurally similar and functionally equivalent.
\end{abstract}

[Key words: unc-55, synaptic specificity, Caenorhabditis elegans, motoneuron, locomotory behavior, GABAergic neurons]

The selection of specific synaptic targets by a neuron represents the completion of a defined sequence of steps in which individual neurons are assembled into a functional neuronal circuit. First, presumptive neurons are generated and positioned; second, the undifferentiated neurons extend processes to appropriate target regions; and finally, synapses are formed. Each step is dependent upon the preceding step and each is influenced to varying degrecs by cell intrinsic and extrinsic events (Goodman

Received Feb. 28, 1994; revised May 31, 1994; accepted July 15, 1994.

This work was supported by the National Science Foundation and the Georgia State University Research Foundation. We are grateful to Mike Nonet for providing the synaptotagmin antiserum and Marty Chalfie, Don Edwards, Paul Linser, Cliff Opdike, Don Wigston, and members of our laboratory for critically reviewing earlier versions of the manuscript. Some of the strains in this work were supplied by the Caenorhabditis elegans Genetics Center, which is funded by the Natiunal Institutes of Health National Center for Research Resources (NCRR)

Correspondence should be addressed to Walter William Walthall at the above address.

Copyright (C) 1995 Society for Neuroscience $0270-6474 / 95 / 151035-09 \$ 05.00 / 0$ and Schatz, 1993). The interdependence between the steps and the inability to distinguish between intrinsic and extrinsic influences contribute to the difficulty in characterizing mechanisms involved in synapse specification.

Animals amenable to genetic manipulations offer the potential to circumvent early steps in the sequence and identify directly genes whose products are involved in synapse specification. The nematode Caenorhabditis elegans is well suited for sophisticated genetic manipulations (Brenner, 1974; Herman, 1986). In addition, a stereotyped pattern of cell divisions produces a nervous system with exactly 302 individually identifiable neurons (Sulston and Horvitz, 1977; Sulston et al., 1983). The associated synaptic patterns that interconnect the neurons and their targets have been determined through reconstructions of serially sectioned animals. These synaptic relationships werc used to assemble a series of circuit diagrams, each underlying a particular behavior (White et al., 1986; Hall and Russell, 1991). The contribution made by individual classes of neurons can be tested by ablating selected cells or their lineal precursors and then comparing the behavior of animals with and without the neurons in question. A variation on this strategy is to examine the defect exhibited by a mutant and then infer from the connectivity diagrams cells likely to be affected. The locomotory circuits in $C$. elegans have been particularly well characterized (White et al., 1976, 1986), and more than 100 different genes have been identified by mutation that cause the animal to become uncoordinated (unc).

Wild-type C. elegans are propelled either forward or backward by sinuous body waves generated by longitudinal dorsal and ventral muscle groups acting antagonistically. In this study we have focused on backward locomotion and the neuronal circuit, derived from White et al. (1986), is diagrammed schematically in Figure $1 B$. Interneurons and motoneurons create contractile waves in which the net magnitude of dorsal and ventral muscular contraction is balanced. Individual classes of motoneurons innervate dorsal or ventral muscle exclusively, therefore it is likely that mutations in genes involved in establishing classspccific synaptic patterns could be identified by selecting mutants in which the balance between dorsal and ventral muscular contraction is lost. Coiling is an example of an unbalanced locomotory pattern and a number of coiling mutants have been isolated. unc-55 mutants, when attempting to move backward, coil with their ventral surfaces oriented centrally; this is operationally defined as a ventral coiler. Here results from genetic, immunohistochemical, and laser ablation experiments show that mutations in the gene $u n c-55$ respecify the synaptic pattern of one inhibitory motoneuron class, causing it to assume the synaptic pattern and function of the other inhibitory class. 


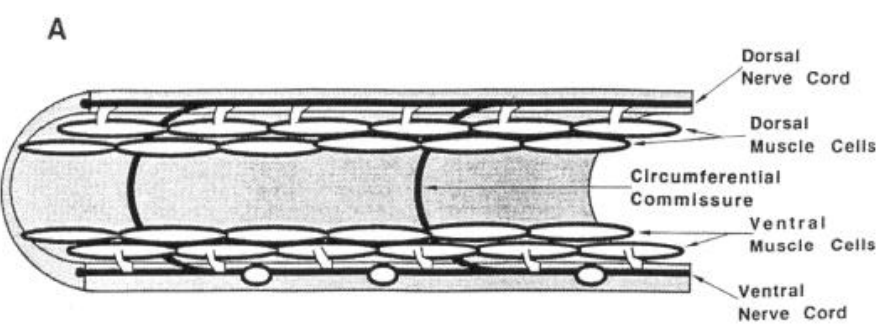

B Cellular Network for Backward Locomotion

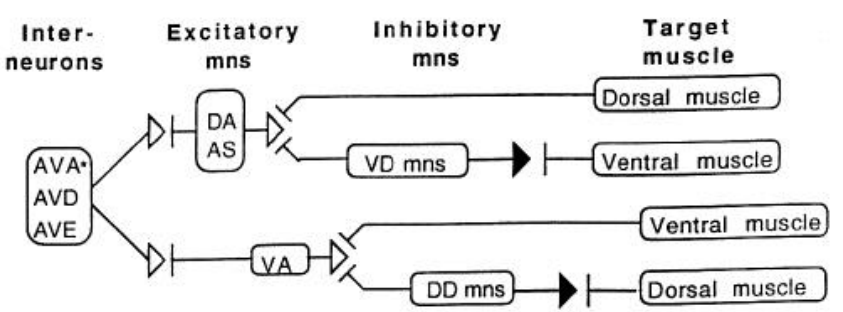

Figure 1. Spatial $(A)$ and synaptic $(B)$ organization of the neurons and muscles responsible for locomotion in $C$. elegans. $A$, The schematic viewed from the left lateral side illustrates the spatial relationship between the dorsal and ventral nerve cords and muscles in the midbody region of $C$. elegans. Muscle arms extend from the bodies of dorsal and ventral muscle to the respective nerve cord where neuromuscular junctions are formed. The open circles represent the positions of motoneuron cell bodies in the ventral nerve cord. A subset of mns have processes in both dorsal and ventral nerve cords; each is connected by circumferential commissure. $B$, Schematic illustration of the neuronal network responsible for backward locomotion in $C$. elegans. Both excitatory (open triangles) and inhibitory (solid triangles) chemical synapses are indicated. Dorsal and ventral muscle being driven by the dorsal excitatory mns are offset from those being driven by the ventral excitatory mns to reflect the antiphasic relationship between dorsal and ventral contractile waves. The asterisk by AVA indicates that it also forms gap junctions onto the DA mns.

\section{Materials and Methods}

\section{Strain maintenance}

In the laboratory, $C$. elegans were kept on petri plates containing nematode growth medium agar (NGM) that had been seeded with Escherichia coli (OP50), the animal's food source (Brenner, 1974). Animals were kept in incubators at 20 or $25^{\circ} \mathrm{C}$. When provided adequate food and not overcrowded, the life cycle is composed of an embryonic developmental stage followed by four larval stages $(\mathrm{Ll}-4)$ before the final molt and emergence of the adult hermaphrodite or male. The entire life cycle is completed in $3.5 \mathrm{~d}$ when animals are kept at $20^{\circ} \mathrm{C}$. The wildtype C. elegans strain N2 and the following mutants were used in this study.

\section{Linkage group I: unc-55 (e402, e1170, and jd6)}

Our experiments have focused primarily on unc-55 (e402). It was generated and characterized by Brenner (1974). Heterozygous unc-55 (e402/ + ) animals exhibit a subtle locomotory defect in which both ventral and dorsal muscular contractions are stronger than normal. Strains homozygous for two additional recessive alleles, $e 1170$ and $j d 6$, were used to confirm structural alterations as well as locomotory changes for each of the reported results. The generation and characterization of additional $u n c-55$ alleles including a description of the likely phenotype of an unc-
55 null allele is currently in progress (C.-Y. Hsu and W. W. Walthall, in preparation).

\section{Linkage group IV: unc-30 (e191)}

Locomotory analysis. Backward locomotion was examined in L4 or young adult hermaphrodites. Individual animals were placed onto lightly seeded NGM plates and locomotion was induced by touching the animal on the head with an eyelash hair that had been glued to a toothpick (Chalfie and Sulston, 1981). Each animal was videotaped and analyzed by tracing its outline onto acetate sheets from individual frames at $1 \mathrm{sec}$ intervals.

Laser ablation. Procedures for identification and laser ablation of selected embryonic precursor cells have been described previously (Avery and Horvitz, 1989; Walthall et al., 1993).

Immunohistochemistry. Fixation, permeabilization, and antisera staining protocols for C. elegans have been described previously (Walthall et al., 1993). Colocalization of synaptotagmin (Nonet et al., 1993) and GABA (Incstar, Stillwater, MN) immunoreactivity was shown using an FITC-labeled anti-mouse IgG to view the synaptotagmin and a rhodamine-labeled anti-rabbit IgG to view the GABA (secondary antibodies were obtained from Boehringer-Mannheim). All of the immunofluorescence micrographs presented are of L4 or young adult animals. An $\mathrm{R} \times \mathrm{C}$ test of independence using the $\mathrm{G}$ test (Sokal and Rohlf, 1969) was used to compare structural features of the VD and DD mns between unc- 55 mutants and wild-type animals.

\section{Results}

C. elegans typically lies on either its right or left side and moves either forward or backward in a sinuous pattern that results from alternate contraction and relaxation of dorsal and ventral muscles (Croll, 1975; Chalfie et al., 1985). There are four longitudinal groups of body muscles: one in each of the two dorsal quadrants acting as a contractile unit, and one in each of the two ventral quadrants acting as a contractile unit. Dorsal and ventral body muscle cells send multiple axon-like extensions, muscle arms, to the dorsal and ventral nerve cords, respectively. These muscle arms receive en passant synapses adjacent to the dorsal and ventral nerve cords from the processes of motoneurons (Fig. 1A). Reconstructions of dorsal and ventral excitatory neuromuscular junctions revealed three synaptic elements: one presynaptic element from the excitatory motoneuron and two postsynaptic elements, one from the muscle arm and a second from one of the two types of D $\mathrm{mn}$. This complex is referred to as a dyad (White et al., 1976). Pharmacological and electrophysiological analyses of mns homologous to the $\mathrm{D}$ mns in Ascaris suum revealed that they are GABAergic and inhibitory (Angstadt et al., 1985; Johnson and Stretton, 1987). D mns are activated in parallel with muscular excitation on one side of the animal and cause the release of GABA onto muscle on the opposite side (Fig. 1B). The six dorsal D (DD) and 13 ventral $\mathrm{D}$ (VD mns) transfer information in opposite directions and compose a cross-inhibitory network primarily involved in maintaining the antiphasic relationship between the contractile waves in dorsal and ventral muscles (McIntire et al., 1993b; Walthall et al., 1993).

\section{Locomotory behavior of unc-55 mutants}

Backward movement of an unc-55 (e402) mutant was videotaped at a magnification of $40 \times$. The left column of Figure 2 shows the sequence of postures that resulted in response to head touch of an unc-55 (e402) animal. The first posture was traced immediately before the initiating touch and subsequent postures were traced at $1 \mathrm{sec}$ intervals. A pronounced imbalance was observed as ventral muscular contractions were stronger than dorsal contractions and resulted in the formation of the ventral coil. The imbalance is likely the result of an alteration that either 


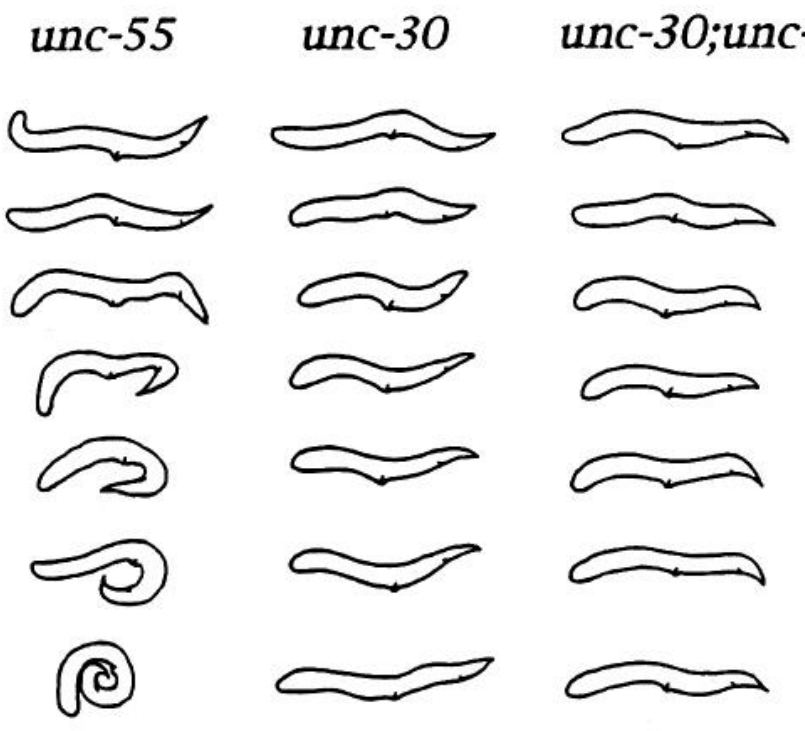

Figure 2. Locomotory patterns exhibited by unc-55, unc-30, and the unc-55; unc-30 double mutant. The top frame in each column represents the animal's postures prior to head touch. Subsequent frames represent postures following the head touch in consecutive $1 \mathrm{sec}$ intervals. During this time the $u n c-55$ mutant traversed a distance of approximately two body lengths, whereas the other two mutants traversed 0.5 body lengths.

decreased excitation onto dorsal muscles or decreased inhibition onto ventral muscles. The VD mns normally inhibit ventral muscles and the possibility that the defect was due to an alteration in ventral inhibition was tested using a mutation that reduces or eliminates inhibition from both of the $\mathrm{D}$ mns. unc30 regulates the differentiation of both the DD and VD mns, and mutations in this gene cause two changes in the D mns: first, the levels of GABA become undetectable using immunohistochemical procedures; and second, an abnormal pattern of synapses was observed at the ultrastructural level (McIntire et al., 1993a). When initiating backward movement, the head and tail of the mutant contract toward the center-the shrinker defect (Fig. 2, central column). Importantly, the magnitude of contraction for the dorsal and ventral muscle was equal and no locomotory imbalance was observed. If the locomotory asymmetry observed in unc-55 is due to a defect in GABA-mediated inhibition from the VD mns, then double mutants homozygous for mutations in unc-30 and unc-55 should exhibit only the locomotory pattern of the unc-30 mutant. Ventral asymmetric locomotion normally exhibited by unc- 55 mutants was completely masked in the presence of the unc-30 mutant (Fig. 2, right column). Thus the ventral asymmetric locomotory pattern exhibited by unc- 55 mutants is dependent upon the presence of functional GABAergic neurotransmission.

In addition to their locomotory defects, unc- 55 males are unable to mate. Presumably, this reflects the absence of a critical contribution made by the VD mns to male mating behavior. To determine whether unc-55 mutants exhibit behavioral defects involving neurons other than the VD mns, various behaviors were compared between unc-55 and wild-type animals. Sensory functions required for mechanosensation (Chalfie and Sulston, 1981), dauer formation (Vowels and Thomas, 1992), and the initiation of pharyngeal pumping (Avery and Horvitz, 1990) were indistinguishable from wild-type animals. The same was true for motor behaviors, such as defecation (Thomas, 1990),
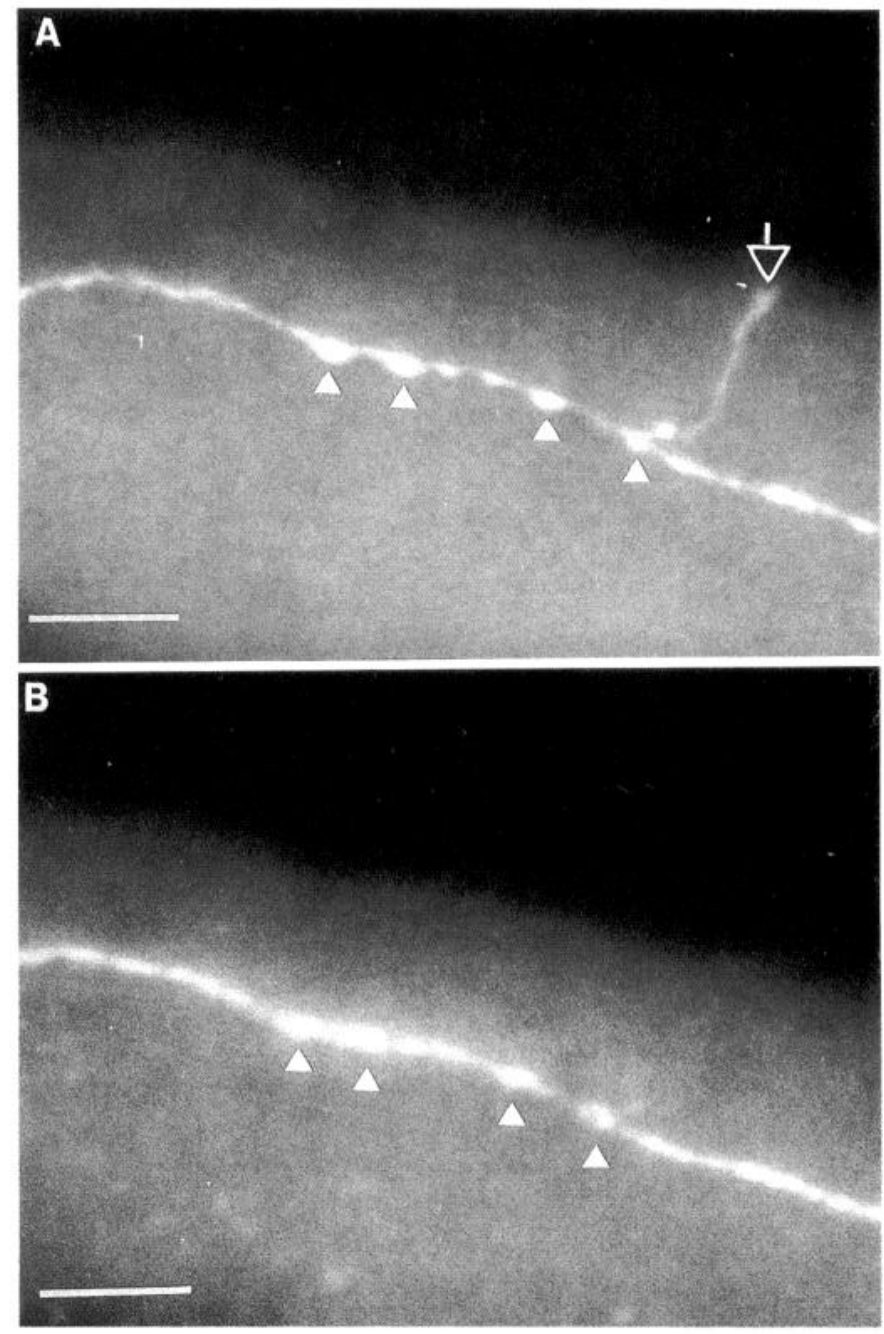

Figure 3. Colocalization of GABA-like and synaptotagmin-like immunoreactivity in the dorsal nerve cord of adult $C$. elegans. Simultaneous double-label immunofluorescence micrographs, viewed from the left side. The four solid arrowheads in each micrograph indicate the same varicosities associated with sites on presynaptic processes. $A$, GABA-like immunoreactivity viewed with rhodamine-tagged secondary antibodies. The open arrow indicates the commissure that connects the dorsal and ventral branches of the D mn. The postsynaptic (VD) and presynaptic processes (DD) of the D mns are the only GABAergic processes in the dorsal nerve cord and they are tightly fasciculated. $B$, Distribution of synaptotagmin-like immunoreactivity (Nonet et al., 1993) viewed with fluorescein-tagged secondary antibodies. Note the absence of the connecting commissure, an indication that synaptotagmin is localized in presynaptic processes within the dorsal nerve cord. Scale bar, $10 \mu \mathrm{m}$.

egg laying (Desai and Horvitz, 1989), and pharyngeal pumping (Avery and Horvitz, 1989).

\section{Immunohistochemical analysis of the $D D$ and $V D$ mns in wild-type and unc-55 animals}

Serial reconstructions using electron micrographs from wildtype animals revealed that the post- and presynaptic processes of the VD and DD mns fasciculate extensively with one another as they travel together in the dorsal and ventral nerve cords (White et al., 1976). These authors also showed that the postsynaptic processes of the D mns maintain a constant diameter, whereas presynaptic processes have prominent varicosities due to the presence of organelles found at presynaptic sites of trans- 
ventral
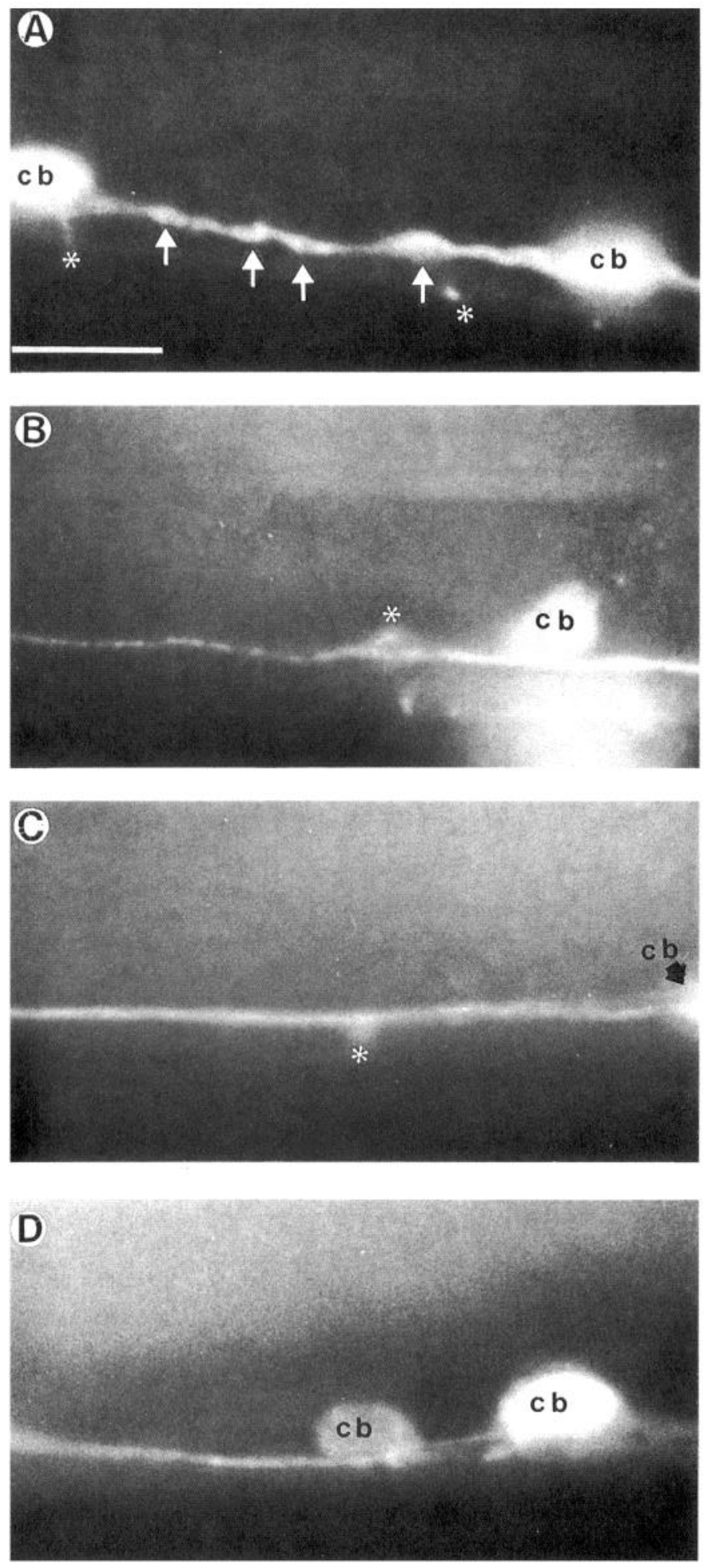

Figure 4. GABA-like immunoreactivity in wild-type $(A)$ and $u n c-55$ animals $(B-D)$. The micrographs in the left column are from the ventral nerve cords, and micrographs in the right column are from the dorsal nerve cords. $A, \mathrm{~N} 2$, mounted with anterior to the left, shows cell bodies (cbs) of VD 6 and VD 7; the solid arrows indicate ventral varicosities. B, unc-55 (e402), mounted with anterior to the left, shows the cb of VD9. C, unc$55(e 1170)$, mounted with anterior to the right, shows the cb of VD 11. D, unc-55 (jd6), mounted with anterior to the right, shows the cbs of VD10 and DD 5. Cell body assignments were made based on McIntire et al. (1992). The asterisks indicate commissures that connect the ventral and dorsal branches of the VD and DD mns. Scale bars, $10 \mu \mathrm{m}$. 
mitter release, such as synaptic vesicles and mitochondria. In addition, unc-104 mutants, which lack the ability to transport synaptic vesicles away from the cell soma, have no synaptic varicosities on presynaptic branches (Hall and Hedgecock, 1991). If varicosities could be detected at the light level and shown to correlate with the presynaptic branches of motoneurons, it would provide a feature that would distinguish postsynaptic processes from presynaptic processes. This would be of particular importance for analyzing the VD mns in unc-55 mutants. To determine whether presynaptic varicosities of the $D$ mns could be detected at the light level we used an immunofluorescence technique to observe $\mathrm{D}$ mn structure in the dorsal and ventral nerve cords.

\section{The wild-type dorsal nerve cord}

The micrograph in Figure $3 A$ shows GABA-like immunoreactivity in the dorsal nerve cord of wild-type animals. The four solid arrowheads indicate GABAergic varicosities. We determined that these varicosities are likely sites of GABA release by staining with antibodies made against the presynaptic, vesicle-associated protein synaptotagmin (Nonet et al., 1993; Fig. $3 B$ ). The arrowheads indicate the same four varicosities shown in Figure $3 A$. The immunoreaclivity of the synaptotagmin is not as discrete as that of GABA, because synaptotagmin is also present in the presynaptic dorsal processes of the excitatory locomotory neurons, which also form synapses in this area. Because the D mns provide the only GABAergic processes in dorsal nerve cord (McIntire et al., 1992), it is likely that these varicosities are associated with presynaptic branches of DD mns.

The postsynaptic processes of the VD mns cannot be distinguished in the presence of the presynaptic processes of the DD mns in the dorsal nerve cord. Laser ablation of the DD mns that allows visualization of the postsynaptic process of the VD mns will be described later.

\section{The wild-type ventral nerve cord}

GABA-like immunoreactivity was detected in four cell types in the ventral nerve cord. These were identified previously as the contiguous processes of the VD and DD $\mathrm{mns}$ and the processes of two unpaired interneuron/motoneurons, AVL and DVB, that run the length of the cord (Fig. 4A; McIntire et al., 1992). Multiple varicosities were detected consistently in these ventral processes (solid arrows in Figs. 4A, 5). Although ultrastructural examination had revealed that neither the AVL nor the DVB process fasciculated with the D mns, it was usually not possible to distinguish among them in the ventral cord using light-level immunohistochemical techniques. However, as described earlier, mutations in the gene $u n c-30(\mathrm{el} / \mathrm{l})$ block the expression of GABA, specifically in the two classes of D mns. In these animals the AVL and DVB processes had been observed in the absence of the $\mathrm{D}$ mns. These neuronal processes maintained a constant caliber in the ventral cord (McIntire et al., 1993b; J. A. Plunkett and W. W. Walthall, unpublished observations). Therefore, we believe that the observed GABAergic varicosities belong to the presynaptic branches of the VD mns. Our attêmpts to colocalize individual GABAergic varicosities with synaptotagmin in the ventral cord were unsuccessful due to the widespread presence of synaptotagmin in the synapses of interneurons and motoneurons.

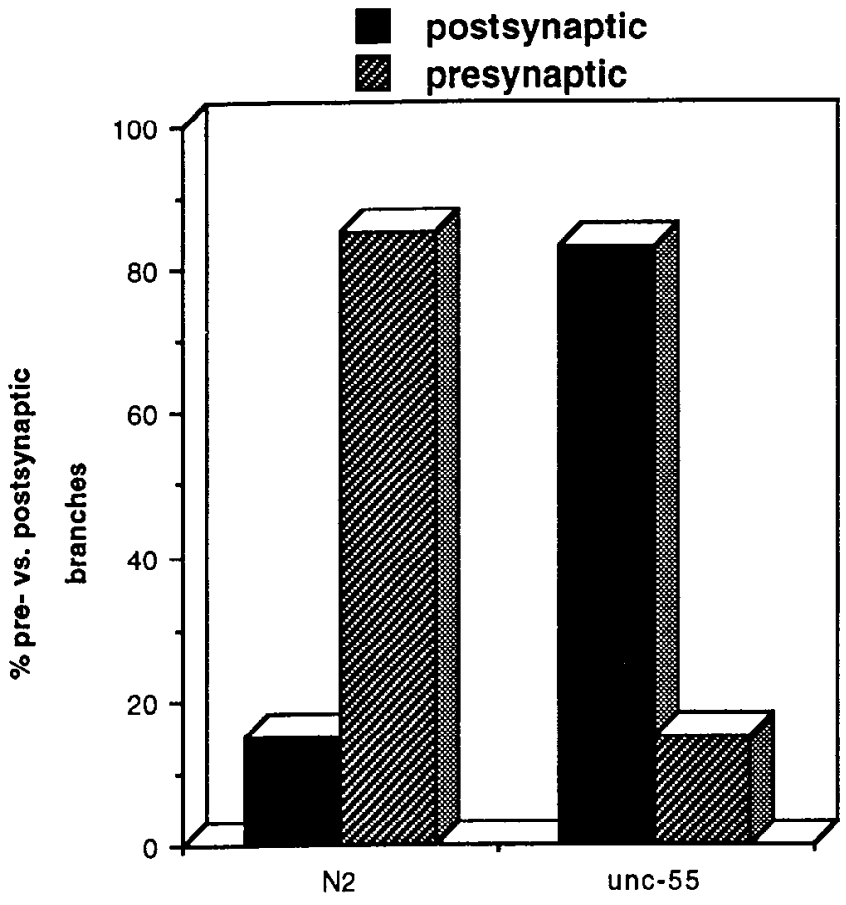

Figure 5. Distribution of pre- and postsynaptic branches in the ventral nerve cord of $\mathrm{N} 2$ and $u n c-55$. The histograms compare the pattern of GABA-likc immunoreactivity as observed in nonoverlapping fields of view for wild-type and $u n c-55$ animals. Varicosities were identified as alterations in the caliber of processes observed in the ventral nerve cord. Branches were designated presynaptic using the criterion of mulliple varicosities and postsynaptic if such varicosities were absent. Although the data were collected primarily from unc-55 (e402), two other unc55 strains were examined ( $e l l 70$ and $j d 6)$. As differences between the groups were negligible, the data from strains homozygous for the three alleles were pooled. See text for a statistical analysis of the data indicating an association between $u n c-55$ mutants and the absence of varicosities.

\section{The ventral cord of unc-55 mutants}

GABA-like immunoreactivity was examined in the ventral cords of three different $u n c-55$ alleles (e402, ell70, and jd6) (Fig. $4 B$ $D$, lcft column). In cach instance the caliber of the $G \Lambda B \wedge$ ergic processes remained constant and small. GABA-like immunoreactivity was observed in the ventral cords of additional $u n c$ 55 and wild-type animals, all of which were either L4 or young adults. The presence of multiple GABAergic varicosities was used as the criterion for concluding that a presynaptic branch was present. Varicosities were observed in $76 \%(123 / 162)$ of the cases in wild-type animals. In contrast, multiple varicosities were observed in $20 \%(19 / 94)$ of the $u n c-55$ group, which was composed of three different alleles: e402 $(n=82)$, e1170 $(n=$ $8)$, and $j d 6(n=4)$. The study focused on unc-55 (e402) primarily, but similar results were obtained from strains homozygous for two other unc-55 alleles and the data from the three strains were pooled. Statistical comparisons were made to determine whether the presence or absence of GABAergic varicosities was correlated with the presence of unc-55 mutations. A $4 \times 2$ frequency table with the four strains forming rows and the presence or absence of varicosities forming the two columns was constructed. There were three degrees of freedom. The calculated $G$-value of 785.612 represented the magnitude of discrepancy between the observed and expected values. It was far greater than the $\chi^{2}$ value $(0.005) 12.838$ and led to the conclusion that an association existed between the absence of ventral GA- 

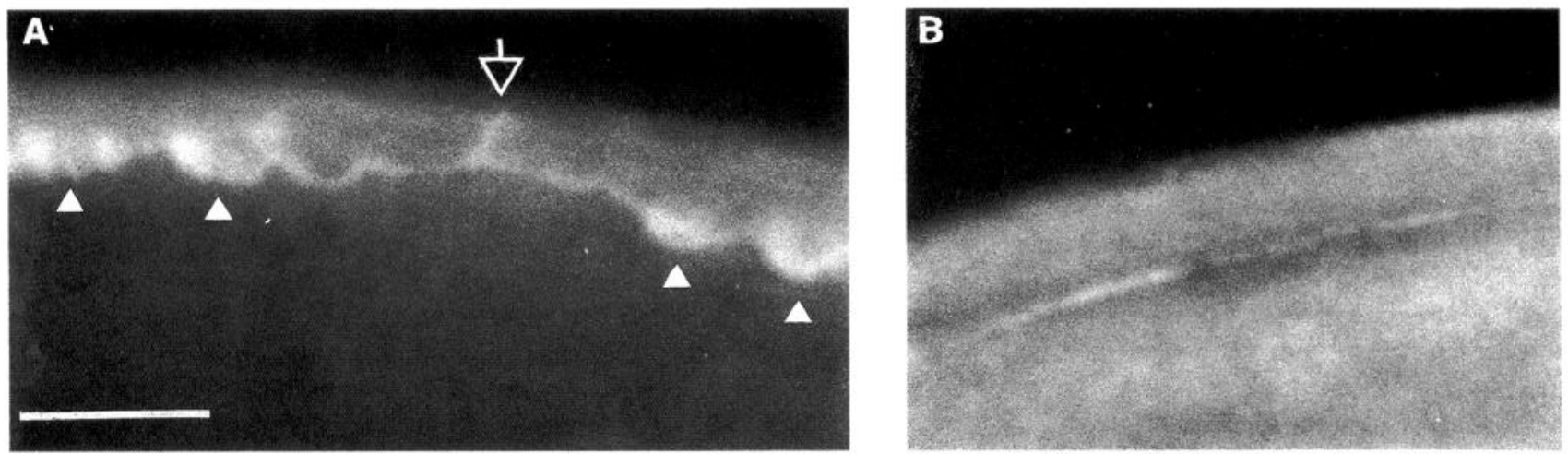

Figure 6. GABA-like immunoreactivity in the dorsal cord of VD mns in DD mn-ablated unc-55 (e402) and wild-type animals. A, An unc-55 (e402) VD mn with commissure (open arrow) has entered the dorsal nerve cord and produced an anterior and posterior branch with varicosities (arrowheads). B, A wild-type, VD mn process in the dorsal nerve cord. VD mn processes were observed in a total of six animals and in each case the dorsal processes, when visible, maintained a constant small caliber (no varicosities) that stained weakly. Scale bar, $10 \mu \mathrm{m}$.

BAergic varicosities and mutations in $u n c-55$. These data are presented graphically in Figure 5.

\section{The dorsal nerve cord of unc-55 mutants}

The presynaptic processes of the DD mns are prominent and fasciculate with the processes of the VD mns (Fig. 4, right column). Therefore, a laser microbeam was used to ablate the two embryonic precursors that produce the six DD mns in unc-55 (e402) mutant embryos (Sulston et al., 1983; Walthall et al., 1993). The absence of the DD mns was confirmed by examining the pattern of $\mathrm{D}$ mn cell bodies of laser-treated and non-lasertreated control $u n c-55$ mutants using GABA immunoreactivity. Nineteen $\mathrm{D}$ mn cell bodies were observed in the mutant ventral nerve cord, but only 13 were present in the ventral cord of the laser-treated mutants; furthermore, cell bodies were absent in each of the locations usually occupied by the six DD mns (data not shown). In the absence of the DD mns the dorsal processes of the unc-55 VD mns showed robust GABA immunoreactivity, and prominent varicosities were present (Fig. 6A, solid arrowheads). This was in sharp contrast to the dorsal processes of VD mns from six wild-type animals. The GABA immunoreactivity was generally weak and the calibers of these processes, when visible, were constant and small (Fig. 6B).

\section{Analysis of the contribution of the transformed unc-55VD mns to locomotion}

These results indicate that the presence of varicosities, a distinctive feature of $C$. elegans presynaptic processes at the ultra- structural level (White et al., 1976), can be reliably observed at the light microscopic level using immunofluorescence. More importantly, we show that the distribution of these varicosities in mutant unc-55 VD mns was reversed from that of wild type. As a result, their ventral processes appeared to be postsynaptic and their dorsal processes appeared to be presynaptic. Whether the transformed VD mns have adopted a function appropriate for their altered synaptic pattern remains to be established. If the function of the VD mns has been blocked in unc- 55 mutants, then the reduction in ventral inhibition would lead to enhanced contraction of ventral muscle and create a ventral asymmetric pattern of locomotion. However, the same asymmetric pattern of locomotion would be expected if the transformed VD mns had adopted the function appropriate for their altered synaptic pattern. In this case the direction of information transfer would be from ventral to dorsal for both classes of $\mathrm{D}$ mns. As a result, dorsal inhibition would be enhanced at the expense of ventral inhibition and would result in an abnormally strong ventral muscular contraction. If $u n c-55 \mathrm{VD}$ mns fail to function, then removal of the DD mns would be expected to produce a shrinker pattern of locomotion, which is the result obtained when VD and DD mn function is disrupted either genetically or surgically (Table 1; McIntire et al., 1993b).

We compared the locomotory patterns of a number of different types of animals in which dorsal muscular inhibition, ventral muscular inhibition, or both were altered either surgically or genetically, and the results are summarized in Table 1. Of particular importance was the comparison of backward lo-

Table 1. Comparison of locomotory patterns with and without functional D mns in wild-type, unc-30, and unc-55 animals.

\begin{tabular}{|c|c|c|c|c|}
\hline Strain & $n$ & $\begin{array}{l}\text { Dorsal muscle } \\
\text { inhibition }\end{array}$ & $\begin{array}{l}\text { Ventral muscle } \\
\text { inhibition }\end{array}$ & $\begin{array}{l}\text { Locomotory } \\
\text { pattern }\end{array}$ \\
\hline Wild-type (N2) & $>100$ & + & + & Balanced \\
\hline unc-30 (e19l) & 20 & - & - & Shrinker \\
\hline unc-55 (e402) & $>100$ & ++ & - & Ventral coiler \\
\hline \multicolumn{5}{|l|}{ unc-55 (e402) } \\
\hline DD ablated & 6 & + & - & Ventral coiler \\
\hline Wild-type & & & & \\
\hline DD ablated ${ }^{a}$ & 30 & - & + & Dorsal coiler \\
\hline
\end{tabular}

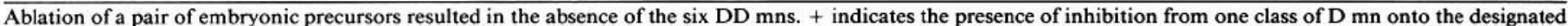
muscle. - indicates the absence of inhibition.

a Twenty-five of the wild-type DD-ablated animals composed the data set for an experiment reported in Walthall et al. (1993). 
comotion in $u n c-55$ mutants and wild-type animals in the absence of the DD mns. The two embryonic precursors that produce the six DD mns were ablated in six unc-55 (e402) mutants, including the one shown in Figure $6 A$. In their absence the unc55 animals coiled ventrally when attempting backward movement (Table 1). In addition to five animals in this study, the DD mns had been ablated previously in 25 wild-type animals and the resulting adults were strong dorsal coilers (Table 1; Walthall et al., 1993). These results suggest that the wild-type VD mns conduct information from the dorsal cord to ventral muscle and the unc-55 VD mns conduct information from the ventral cord to dorsal muscle.

In summary, the immunohistochemical results and the asymmetric pattern of locomotion in DD-ablated unc-55 mutants suggest that the VD mns have adopted the synaptic pattern and function of the wild-type DD mns. Thus, mutating a single gene caused the VD mns to adopt the synaptic pattern and function of the functionally related DD mns.

\section{Discussion}

Our goal is to investigate underlying genetic programs that allow neurons to establish appropriate synaptic patterns. $C$. elegans has an experimentally tractable genetic system and a nervous system in which defective synaptic patterns can be detected. Two stereotyped locomotory patterns (forward and backward) are produced in the body of $C$. elegans by seven classes of motoneurons (Chalfie et al., 1985). Importantly, the interconnecting synaptic patterns have been determined (White et al., 1976, 1986) and the specific contribution made by each class of locomotory neuron has been defined either explicitly, based on laser ablation studies (Chalfie et al., 1985; McIntire et al., 1993b; Walthall et al., 1993), or implicitly, based on synaptic patterns (White et al., 1986). The hierarchical organization of the neuronal network that generates backward locomotion is illustrated in Figure $1 B$. Three pairs of locomotory interneurons devoted to backward locomotion inncrvate threc classes of excitatory motoneurons, two (DA and AS) that innervate dorsal muscles and one (VA) that innervates ventral muscles. The two inhibitory $m n$ classes, DD and VD, form a cross-inhibitory network that insures that muscular contraction on one side is complemented by muscular relaxation on the opposite side. As a result, metachronous, oscillatory waves of dorsal and ventral muscular contraction maintain a constant phase relationship as they pass anteriorly along the animal's longitudinal axis during backward movement (Chalfie et al., 1985).

The importance of maintaining the proper balance between dorsal and ventral muscular inhibition and excitation has been demonstrated for locomotion using both surgical and genetic manipulations. In the absence of the six DD mns, wild-type animals (L4 or young adult) produce a dorsal asymmetric locomotory pattern when moving backward (Walthall et al., 1993). The imbalance resulted from the absence of DD mn-mediated dorsal inhibition and the presence of VD mn-mediated ventral inhibition. Dorsal asymmetric locomotory patterns also result from mutations that decrease ventral excitation. In $u n c-4 \mathrm{mu}-$ tants the complement of backward locomotory interneurons form appropriate connections with the DA but not the VA mns (see Fig. $1 B$ ). The dorsal locomotory asymmetry results from activation of the motoneurons that cause dorsal muscular contraction in the absence of ventral muscular contraction.

The synaptic input of nine of the 12 VA mns is affected by the $u n c-4$ mutation. The lineages that produce these nine VA mns also produce sibling VB mns (normally the VB mns drive ventral muscle during forward movement) (Sulston and Horvitz, 1977). The interneuronal innervation of the three VA mns that do not have sibling VB mns is correct (White et al., 1992). In unc- 4 mutants the VB and VA mns are both innervated by a pair of interneurons dedicated to forward movement. unc-4 possesses a homeobox and is expressed in both the DA and VA mns (Miller et al., 1992, 1993); apparently this gene regulates the transcription of genes in the VA mns whose products allow interneurons involved in backward and forward movement to distinguish between sibling VA and VB mns.

Results presented here suggest that the ventral coiling defect exhibited by $u n c-55$ mutants is due to specific alterations in the afferent and efferent synapses associated with the VD mns. An imbalance is created between dorsal and ventral muscular contractions due to the coincidental subtraction of ventral inhibition and addition of dorsal inhibition, as illustrated in Figure 7. Both changes contribute to the asymmetric ventral coiling defect. In essence, the VD mns have adopted the structure and function of the DD mns. The degree of this transformation varies in an allele-specific manner among strains homozygous for different $u n c-55$ alleles (Hsu and Walthall, in preparation).

It is surprising that mutations in one gene, unc-55, would alter both the afferent and efferent synaptic patterns made by an entire class of $\mathrm{mn}$. It is, perhaps, more surprising that these mutations cause the VD mns to adopt the synaptic pattern and function of the DD mns. This result provides insight into the genetic specification of the two classes of D mns. At least 12 genes, when mutated, cause identical defects in both classes. These mutations have identified genes involved in both the structural (Hedgecock et al., 1990; McIntire et al., 1992) and functional differentiation (McIntire et al., 1993a) of the D mns. Their structural differentiation is dependent upon separate longitudinal and circumferential coordinate systems that are used by a number of migratory cells and cellular processes (Hedgecock et al., 1990; McIntire et al., 1992). The molecular analysis of two genes involved in circumferential guidance revealed molecules that share similarities with a component of the vertebrate extracellular matrix (laminin) or matrix receptors (immunoglobulin and thrombospondin domains) (Ishii et al., 1992; LeungHagesteijn et al., 1992). Both the VD and the DD mns are able to utilize independently this coordinate system for process guidance (Walthall et al., 1993).

Both $\mathrm{D}$ mns receive cholinergic input from excitatory mns onto their postsynaptic processes and both release the neurotransmitter GABA from their presynaptic processes. Mutations in five genes have been characterized that disrupt GABAergic neurotransmission (McIntire et al., 1993a,b). These mutants shrink when the animal reverses its direction of movement due to the simultaneous contraction of diametrically opposed dorsal and ventral muscles. Although the phase relationship between dorsal and ventral muscular contractions is disrupted in these mutants, no imbalance is created because both dorsal and ventral inhibition is affected equally. One of the mutations, unc49 , identified a gene that encodes the $\mathrm{GABA}_{\mathrm{A}}$ receptor on the muscle (Mancillas et al., 1991; McIntire et al., 1993b). Three of the shrinker mutations, unc-25, unc-46, and unc-47, represent genes involved in the general synthesis and release of GABA from the presynaptic process of the D mns (McIntire, 1993a). The phenotypes of these three mutants, in addition to disrupting locomotion, alter foraging and defecation, behaviors dependent on other GABAergic motoneurons. A fifth gene, unc-30, plays 

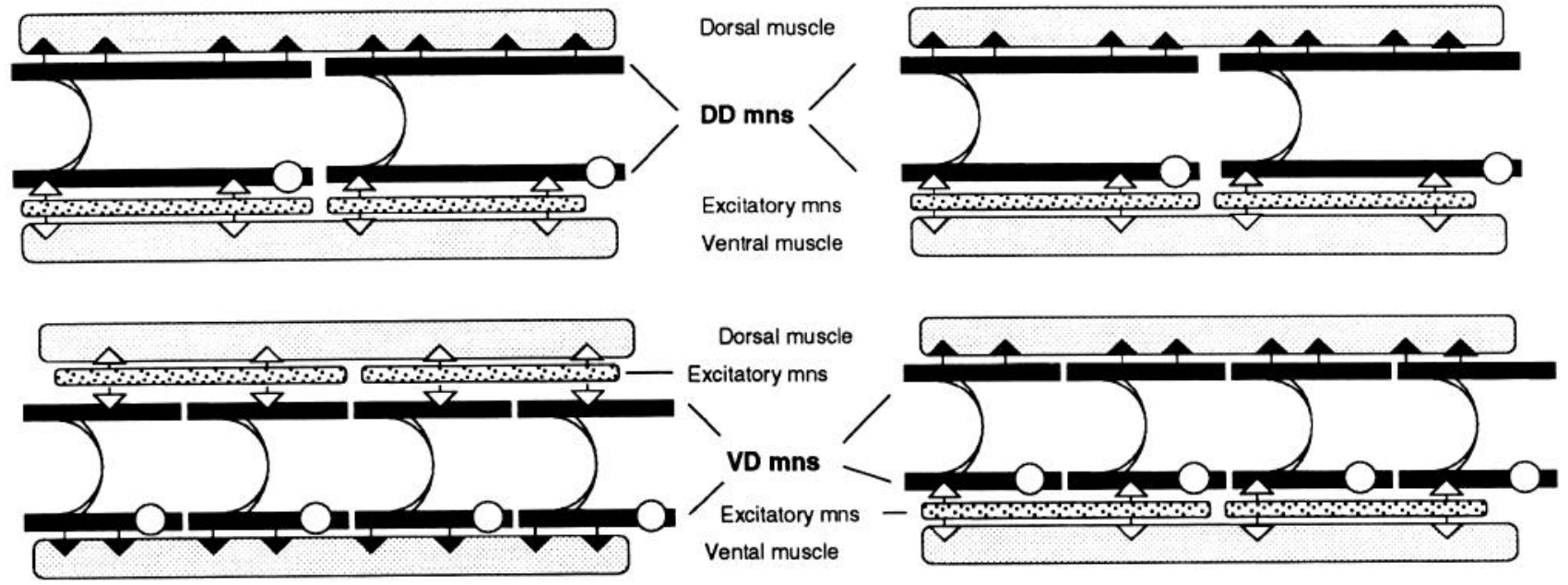

Figure 7. The synaptic patterns of the DD and VD mns in wild-type and unc-55 animals. These schematic diagrams are drawn as viewed from the left lateral perspective. The top left illustration shows the presynaptic process of the DD $\mathrm{mn}$ in the dorsal nerve cord connected via a circumferentially running commissure to the postsynaptic process and cell body (open circle) in the ventral nerve cord; thus this class of neuron conducts information from ventral to dorsal. Each of the four VD mns illustrated possess a postsynaptic process in the dorsal nerve cord that is connected via a circumferentially running commissure to a presynaptic process and cell body (open circle) in the ventral nerve cord; thus this class of neuron conducts information from dorsal to ventral. The schematics on the right represent the pattern of synapses for the DD and VD mns in unc-55 mutants. The synaptic pattern for the DD mns remains unchanged; however, the VD mns have adopted a synaptic pattern identical to that of the DD mns. Inhibitory neuromuscular junctions are indicated by filled arrows and excitatory synapses by open arrows. Other processes in both cords were excluded for simplicity.

a specific regulatory role in the differentiation of the $\mathrm{D}$ mns. Behaviors dependent upon the other GABAergic motoneurons function normally in unc-30 mutants (McIntire et al., 1993a).

The analysis of genes involved specifically in determining synaptic specificity is rather limited. VanVactor et al. (1993) isolated a series of mutants in Drosophila that formed a genetic hierarchy that directs a corresponding hierarchy of cellular events associated with the innervation of segmentally repeated abdominal muscles. Mutations were described that interfere with process guidance, as well as two aspects of target recognition: first, the exploration of subsets of neighboring muscles, termed "target domains," and second, the selection of appropriate subsets of muscle from these domains. The genes identified by mutations acting late, both in the genetic and cellular hierarchy, appear to generally decrease the target selectivity of the developing motoneurons. In both $C$. elegans and Drosophila, genetic and cellular hierarchies exist for directing morphological differentiation and synapse specification. The analysis of synaptic patterns in $u n c-55$ and $u n c-4$ mutants revealed that the affected neurons had adopted synaptic specificities normally reserved for classes of neurons related either by function, in the case of unc-55, or by lineage, in the case of unc-4. The number of genes involved in the differentiation of both $\mathrm{D}$ mn classes leads us to speculate that during embryogenesis the " $\mathrm{D} \mathrm{mn}$ genetic program" is expressed and produces the six DD mns; later, after hatching, the same genetic program is resurrected to produce the 13 VD mns. unc-55 then acts to determine the synaptic pattern that distinguishes the VD from the DD mns. Mutations in $u n c-55$ cause the VD mns to adopt the synaptic pattern and function of the DD mns.

Synaptic specification represents a critical phenotypic feature of neurons. The alteration of VD mn synaptic specificity in unc55 mutants represents a homeotic transformation of the VD cell fate. Investigations of homeotic transformations in Drosophila have identified two gene families involved in specifying cellular fates, the homeobox genes that are involved in establishing the longitudinal axis of virtually all metazoans (Wang et al., 1993), and the EGF gene family, which plays an important role in mediating influential cellular interactions in a number of different species (Greenwald et al., 1983; Greenwald and Seydoux, 1990). The tremendous amount of synaptic diversity generated in mature nervous systems creates a paradox: how does a finite amount of genetic material specify the synaptic complexity necessary to construct even the simplest nervous system? Further analysis of unc-55 should provide insight into genetic mechanisms that influence synaptic specificity among the D mns and could potentially reveal universal strategies for generating synaptic diversity among genetically similar sets of neurons.

\section{References}

Avery L, Horvitz HR (1989) Pharyngeal pumping continues after laser killing of the pharyngeal nervous system of Caenorhabditis elegans. Neuron 3:473-485.

Avery L, Horvitz HR (1990) Effects of starvation and neuroactive drugs on feeding in Caenorhabditis elegans. J Exp Zool 253:263-270.

Brenner S (1974) The genetics of Caenorhabditis elegans. Genetics 77: 71-94.

Chalfie M, Sulston JE (1981) Developmental genetics of the mechanosensory neurons of Caenorhabditis elegans. Dev Biol 82:358-370.

Chalfie M, Sulston JE, White JG, Southgate E, Thomson JN, Brenner S (1985) The neural circuit for touch sensitivity in Caenorhabditis elegans. J Neurosci 5:956-964.

Croll NA (1975) Components and patterns in the behavior of the nematode Caenorhabditis elegans. J Zool 176:159-176.

Desai C, Horvitz HR (1989) Caenorhabditis elegans mutants defective in the functioning of the motor neurons responsible for egg laying. Genetics 121:703-721.

Goodman CS, Schatz CJ (1993) Developmental mechanisms that gen- 
erate precise patterns of neuronal connectivity. Cell 72/Neuron 10 : $77-98$.

Greenwald IS, Seydoux G (1990) Analysis of gain-of-function mutations of the lin-12 gene of Caenorhabditis elegans. Nature 346:197199.

Greenwald IS, Sternberg PW, Horvitz RH (1983) The lin-12 locus specifies cell fates in Caenorhabditis elegans. Cell 34:435-444.

Hall DH, Hedgecock EM (1991) Kinesin-related gene unc-104 is required for axonal transport of synaptic vesicles in C. elegans. Cell 65: 837-847.

Hall DH, Russell RL (1991) The posterior nervous system of the nematode Caenorhabditis elegans: serial reconstruction of identified neurons and complete pattern of synaptic interactions. J Neurosci 11: $1-22$.

Hedgecock EM, Culotti JG, Hall DH (1990) The unc-5, unc-6, and unc- 40 genes guide circumferential migrations of pioneer axons and mesodermal cells on the epidermis in C. elegans. Neuron 4:61-85.

Herman RK (1986) Genetics. In: The nematode Caenorhabditis elegans (Wood WB, ed), pp 17-46. Cold Spring Harbor, NY: Cold Spring Harbor Laboratory.

Ishii N, Wadsworth WG, Stern BD, Culotti JG, Hedgecock EM (1992) $U N C$-6, a laminin-related protein, guides cell and pioneer axon migrations in C. elegans. Neuron 9:873-881.

Johnson CD, Stretton AOW (1987) GABA-immunoreactivity in inhibitory motor neurons of the nematode Ascaris. J Neurosci 7:223235.

Leung-Hagesteijn C, Spence AM, Stern BD, Zhou Y, Su MW, Hedgecock EM, Culotti JG (1992) UNC-5, a transmembrane protein with immunoglobulin and thrombospondin type 1 domains, guides cell and pioneer axon migrations in C. elegans. Cell 71:289-299.

Mancillas JR, Ruiz-Morales A, Olsen R (1991) GABA receptor function is altered in the $C$. elegans mutant unc-49. Soc Neurosci Abstr 17:526.

McIntire SL, Garriga G, White JG, Jacobson D, Horvitz HR (1992) Genes necessary for directed axonal elongation or fasciculation in $C$. elegans. Neuron 8:307-322.

McIntire SL, Jorgensen E, Horvitz HR (1993a) Genes required for GABA function in Caenorhabditis elegans. Nature 364:334-337.

McIntire SL, Jorgensen E, Kaplan J, Horvitz HR (1993b) The GABAergic nervous system of Caenorhabditis elegans. Nature 364:337341.

Miller DM, Shen MM, Shamu CE, Burglin TR, Ruvkun GR, Dubois ML, Ghee M, Wilson L (1992) C. elegans unc-4 gene encodes a homeodomain that determines the pattern of synaptic input to specific motor neurons. Nature 355:841-845.
Miller DM, Niemeyer CJ, Chitkara P (1993) Dominant unc-37 mutations suppress the movement defect of a homeodomain mutation in $u n c-4$, a neural specificity gene in Caenorhabditis elegans. Genetics 135:741-753.

Nonet ML, Grundahl K, Meyer BJ, Rand JB (1993) Synaptic function is impaired but not eliminated in $C$. elegans mutants lacking synaptotagmin. Cell 73:1291-1305.

Salser S, Loer C, Kenyon C (1993) Multiple Hom-C interactions specify cell fates in the nematode central nervous system. Genes Dev 7:1714-1724.

Sokal RR, Rohlf FJ (1969) Biometry: the principles and practice of statistics in biological research. San Francisco: Freeman.

Sulston JE, Horvitz HR (1977) Postembryonic cell lineages of the nematode Caenorhabditis elegans. Dev Biol 56:110-156.

Sulston JE, Schierenberg E, White JG, Thomson JN (1983) The embryonic cell lineage of the nematode Caenorhabditis elegans. Dev Biol 100:64-119.

Thomas JH (1990) Genetic analysis of defecation in Caenorhabditis elegans. Genetics 124:855-872.

VanVactor D, Sink H, Fambrough D, Tsoo R, Goodman CS (1993) Genes that control neuromuscular specificity in Drosophila. Cell 73: $1137-1153$.

Vowels JJ, Thomas JH (1992) Genetic analysis of chemosensory control of dauer formation in Caenorhabditis elegans. Genetics 130:105123.

Walrond JP, Kass I, Stretton AOW (1985) Reciprocal inhibition in the motor nervous system of the nematode Ascaris: direct control of ventral inhibitory motoneurons by dorsal excitatory motoneurons. $\mathrm{J}$ Neurosci 5:9-15.

Walthall WW, Li L, Plunkett JA, Hsu C-Y (1993) Changing synaptic specificities in the nervous system of Caenorhabditis elegans: differentiation of the DD motoneurons. J Neurobiol 24:1589-1599.

Wang BM, Muller-Immergluck M, Austin J, Robinson A, Chisolm A, Kenyon C (1993) A homeotic gene cluster patterns the anteroposterior body axis of $C$. elegans. Cell 74:29-42.

White JG, Southgate E, Thomson JN, Brenner S (1976) The structure of the ventral nerve cord of Caenorhabditis elegans. Philos Trans $\mathrm{R}$ Soc Lond [Biol] 275:327-348.

White JG, Southgate E, Thomson JN, Brenner S (1986) The structure of the nervous system of the nematode Caenorhabditis elegans. Philos Trans R Soc Lond [Biol] 314:1-340.

White JG, Southgate E, Thomson JN (1992) Mutations in the Caenorhabditis elegans unc-4 gene alter the synaptic input to ventral cord motor neurons. Nature 355:838-841. 